\title{
Differences in time course activation of dorsolateral prefrontal cortex associated with low or high risk choices in a gambling task
}

\section{Stefano Bembich ${ }^{1}$, Andrea Clarici ${ }^{2 *}{ }^{*}$, Cristina Vecchiet ${ }^{1}$, Giulio Baldassi ${ }^{1}$, Gabriele Cont ${ }^{1}$ and Sergio Demarini ${ }^{1}$}

1 Institute for Maternal and Child Health, IRCCS "Burlo Garofolo," Trieste, Italy

${ }^{2}$ Psychiatric Clinic Unit, Department of Medical, Surgical and Health Sciences, University of Trieste, Trieste, Italy

\section{Edited by:}

John J. Foxe, Albert Einstein College of Medicine, USA

Reviewed by:

Ippeita Dan, Jichi Medical

University, Japan

Colm Gerard Connolly, University of

California San Francisco, USA

*Correspondence:

Andrea Clarici, Psychiatric Clinic

Unit, Department of Medical,

Surgical and Health Science,

University of Trieste, Via Paolo de

Ralli 5, 34138 Trieste, Italy

e-mail: clarici@units.it
Prefrontal cortex plays an important role in decision making (DM), supporting choices in the ordinary uncertainty of everyday life. To assess DM in an unpredictable situation, a playing card task, such as the lowa Gambling Task (IGT), has been proposed. This task is supposed to specifically test emotion-based learning, linked to the integrity of the ventromedial prefrontal cortex (VMPFC). However, the dorsolateral prefrontal cortex (DLPFC) has demonstrated a role in IGT performance too. Our aim was to study, by multichannel near-infrared spectroscopy, the contribution of DLPFC to the IGT execution over time. We tested the hypothesis that low and high risk choices would differentially activate DLPFC, as IGT execution progressed. We enrolled 11 healthy adults. To identify DLPFC activation associated with IGT choices, we compared regional differences in oxy-hemoglobin variation, from baseline to the event. The time course of task execution was divided in four periods, each one consisting of 25 choices, and DLPFC activation was distinctly analyzed for low and high risk choices in each period. We found different time courses in DLPFC activation, associated with low or high risk choices. During the first period, a significant DLPFC activation emerged with low risk choices, whereas, during the second period, we found a cortical activation with high risk choices. Then, DLPFC activation decreased to non-significant levels during the third and fourth period. This study shows that DLPFC involvement in IGT execution is differentiated over time and according to choice risk level. DLPFC is activated only in the first half of the task, earlier by low risk and later by high risk choices. We speculate that DLPFC may sustain initial and more cognitive functions, such as attention shifting and response inhibition. The lack of DLPFC activation, as the task progresses, may be due to VMPFC activation, not detectable by fNIRS, which takes over the IGT execution in its second half.

Keywords: multichannel NIRS, DLPFC, risk, lowa Gambling Task, attention shifting, response inhibition

\section{INTRODUCTION}

There is a general agreement in the role of the prefrontal cortex in decision-making (DM), under conditions of complexity and unpredictability (e.g., Bechara et al., 2000a; Bechara, 2001; Clark et al., 2003; Wise, 2008; Gläscher et al., 2012). According to Damasio's "somatic marker hypothesis" (Damasio, 1994), patients with ventromedial prefrontal cortex (VMPFC) damage develop severe impairments in personal and social DM, while other intellectual abilities are usually well preserved. There appears to be an important deficit in the emotional learning process: the somatic activation of an emotional response is no longer associated with DM, in uncertain conditions, therefore impairing the DM process itself.

In order to assess the complex nature of human DM under uncertainty, the Iowa Gambling Task (IGT) has been developed (Bechara et al., 1994). It is a playing card task which simulates real-life decisions. The IGT is supposed to specifically test emotion-based learning, linked to DM under uncertainty. For example, during the task, participants show an anticipatory skin conductance response associated with risky choices (Bechara et al., 1999), while patients with a damage in VMPFC, both fail the test and do not show anticipatory skin conductance responses (Bechara et al., 1994, 2000b). Additionally, VMPFC may play a role in the continuous updating of expectations about reward and punishment based on experience (Fellows and Farah, 2005; Oya et al., 2005; Stocco and Fum, 2007).

Other areas of the prefrontal cortex may play a relevant role in DM processes associated with the IGT execution. Among these, the most important seems to be the dorsolateral prefrontal cortex (DLPFC), whose role in a gambling task has been demonstrated both by clinical and neuroimaging studies. Patients with a lesion in the right DLPFC showed significantly worse performances than patients with focal lesions in other areas (with the exclusion of VMPFC) or controls (e.g., Manes et al., 2002; Clark et al., 2003). 
Moreover, Fellows and Farah (2005) found that eliminating the need for a reversal learning from IGT improved the performance of VMPFC patients, but not that of DLPFC patients. In addition, using positron emission tomography (PET), a significant activation in right DLPFC was found during IGT execution (e.g., Ernst et al., 2002, 2003; Bolla et al., 2003, 2005).

In a recent functional magnetic resonance imaging (fMRI) research, a modified double deck version of IGT was used to study the role of prefrontal cortex in task rule learning (Hartstra et al., 2010). A differential involvement of DLPFC, anterior cingulated cortex (ACC)/pre-supplementary motor area (pre-SMA) and medial orbital frontal cortex (med-OFC) was observed, as IGT execution progressed. DLPFC and ACC/pre-SMA were more active during the first phase of IGT performance, while medOFC was more active in later phases. However, differential cortical activation according to different choice risk levels was not studied.

So far IGT has been studied only by fMRI (e.g., Lin et al., 2008; Lawrence et al., 2009) or PET (e.g., Ernst et al., 2002). Consequently, the execution of the original task had to be adapted to the spatial and technical limitations of such devices (e.g., implementing a computerized version of IGT or limiting cerebral activity detection to a restricted part of the test). As a possible alternative, closer to real life situations, multichannel near-infrared spectroscopy (NIRS) may be used (e.g., Cazzell et al., 2012). It permits detection of regional hemoglobin concentration changes in the cortex induced by brain activity with fairly good spatial resolution and very high temporal resolution (Maki et al., 1995; Taga et al., 2003), even if detection is limited to the cortical surface. NIRS cortical monitoring is non-invasive, does not require strict motion restriction during measurements (subjects are not put into a scanner), is safe (Ito et al., 2000) and resistant to movement artifacts. These characteristics make it particularly suitable for research concerning also complex cognitive functions with awake healthy subjects in a relatively unrestricted environment.

Although DLPFC activation during IGT has been observed mainly in the initial phases of the performance (Hartstra et al., 2010), there are no studies assessing if such activation is differentially associated with high- or low-risk choices as the task goes on. The aim of this pilot exploratory study was to further assess, by multichannel NIRS, the dynamic contribution of DLPFC to the IGT execution over time, in healthy adults. We tested the hypothesis that DLPFC would be differentially activated by low- and high-risk choices as the IGT execution progressed.

\section{MATERIALS AND METHODS PARTICIPANTS}

A total of 11 participants (seven females), aged between 21 and 38 years (mean: $27.7 \pm 5.7$ ), participated to this study. They were all healthy and there was no history of neurological, sensory, psychiatric or addictive disorders. All were right handed, as assessed by the Edinburgh Questionnaire (Oldfield, 1971). Written informed consent was obtained from each participant after full procedural and technical explanation of the experiment. The ethics committee of the Institute for Maternal and Child Health IRCCS "Burlo Garofolo"-Trieste (Italy), where all tests were conducted, approved the research.

\section{THE IOWA GAMBLING TASK}

We administered the IGT as proposed in the original study of Bechara et al. (1994), using physical playing cards. Participants chose 100 cards from four decks (A, B, C, D) in any sequence they preferred. There were two advantageous or low risk decks $(\mathrm{C}$ and $\mathrm{D})$, and two disadvantageous or high risk decks (A and B). The schedule of rewards and punishments differed in decks: in deck A (disadvantageous/high risk) and deck C (advantageous/low risk) there were smaller but more frequent unpredicted punishments, while in deck B (disadvantageous/high risk) and deck D (advantageous/low risk) punishments were greater but less frequent. The only difference from the original task consisted in the value of rewards and punishments and in the currency (Euros rather than Dollars). Participants were actually given a total amount of $€ 2000$ (fake money) at the beginning of the task. When playing on disadvantageous decks, participants won $€ 100$ for every card turned, but incurred in losses between $€ 150$ and $€ 1250$. When playing with advantageous decks, they won $€ 50$ for every card turned and incurred in losses between $€ 25$ and $€ 250$. If subjects chose all the cards in decks A and B they lost comprehensively $€ 1000$, if they chose all the cards in decks C and D they won comprehensively $€ 1000$. Every win or loss actually modified the amount of money held by the participant, and he/she was invited to consider the amount of fake money possessed, won or lost as real and own money. The aim of the game was to win as much money as possible, and to avoid disadvantageous decks. A total net score of less than 50 advantageous choices is the cutoff value that indicates that participants are not choosing cards advantageously.

\section{MULTICHANNEL NIRS RECORDING}

In order to detect cortical activity, we used the Hitachi ETG100 OT device (Hitachi Medical Corporation, Tokyo, Japan), which is a multichannel NIRS system (Maki et al., 1995) recording simultaneously from 24 channels on the cortex. It emits near-infrared light at two wavelengths, 780 and $830 \mathrm{~nm}$, and the reflected light is sampled once every $100 \mathrm{~ms}$. This device estimates changes in the concentration of oxy-hemoglobin (HbO2) deoxyhemoglobin and total hemoglobin in response to stimulation in the unit of $\mathrm{mM} \bullet \mathrm{mm}$, that is the product of the hemoglobin concentration changes expressed in millimolar and the optical path length expressed in millimeters.

For detecting DLPFC activation during the IGT, we used a $4 \times 4$ plastic fibers holder containing 16 optical fibers (or optodes) of $1 \mathrm{~mm}$ in diameter, 8 emitters and 8 detectors, which were placed $3 \mathrm{~cm}$ apart and allowed 24 detecting channels. The fibers holder was positioned above the frontal lobes of participants using the international 10-20 EEG placement system (Jasper, 1958). The anterior row of channels (Figure 1) was put on the virtual line joining Fp1 and Fp2 points, placing channel 23 on Fpz. When the holder was positioned, it was made sure that the fibers touched the scalp. The device automatically detects whether the contact is adequate to measure emerging photons.

Cortical areas covered by each channel were identified referring to the methods developed by Tsuzuki et al. (2007) and by Singh et al. (2005), which allow fNIRS data to be probabilistically registered to the standard Montreal Neurological 


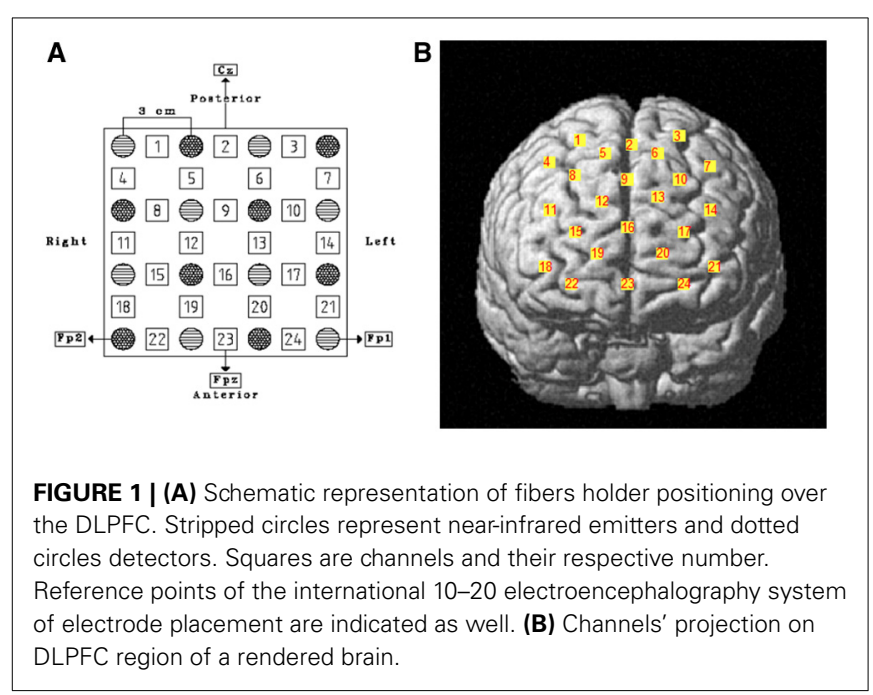

Institute (MNI) space also without using a 3D digitizer, as in our case. The probabilistic localization for each channel is given in Table 1. Cerebral labeling is based on the brain atlas constructed by Tzourio-Mazoyer et al. (2002). To have an estimate of channel projections on a rendered brain, we used the "Spatial registration of NIRS channels location" function of the NIRSSPM version 4_r1 software (Ye et al., 2009), which is a SPM5 and MATLAB based software package (http://bisp.kaist.ac.kr/ NIRS-SPM). Using the "Stand alone" option, not needing MRI images, we obtained a spatial representation of the channel locations on a rendered brain, referring to the MNI coordinates reported in Table 1.

\section{PROCEDURE}

We administered the IGT in a soft lighted and sound isolated room, and participants sat on a chair in front of a desk. In normal adults, the cerebral vascular response takes about 10-12 s to be completed (Wobst et al., 2001; Meek, 2002). Thus, we adjusted the IGT test to detect DLPFC activation associated to each choice: approximately $12 \mathrm{~s}$ were allowed between choosing cards. Specifically, the participant could make each choice only after the presentation of a green slide on a computer screen, which was positioned in front of him or her on the same table where the IGT was performed, behind the four card packs. The slide was presented for $6 \mathrm{~s}$, then there was a break of other $6 \mathrm{~s}$, when the computer screen turned black. Then, the green slide was shown again, for the successive IGT choice and so it went on for the 100 picks of the entire DM task (Figure 2). We hypothesized that, separating each choice from the following one by $12 \mathrm{~s}$, it would allow us to assess the DLPFC activation associated with each choice (Schroeter et al., 2004), with a limited interference on the IGT learning processes (Gupta et al., 2009). The entire experimental procedure was completed in about $25 \mathrm{~min}$.

\section{DATA ANALYSIS}

Our analyses focused on the increase of $\mathrm{HbO} 2$ concentration, which is considered an estimate of cerebral activation (e.g., Meek, 2002). Possible components of the $\mathrm{HbO} 2$ signal related to slow fluctuations of cerebral blood flow and heartbeat noise were
Table 1 | Probabilistic cortical channels localization in MNI space and the corresponding cortical labeling.

\begin{tabular}{|c|c|c|c|c|c|}
\hline \multirow[t]{2}{*}{ Channel } & \multirow[t]{2}{*}{ Anatomical label } & \multicolumn{4}{|c|}{$\begin{array}{l}\text { MNI coordinates } \\
\text { estimation }(\mathrm{mm})\end{array}$} \\
\hline & & $\boldsymbol{x}$ & $y$ & $z$ & $S D$ \\
\hline 1 & R F sup gyrus & 24 & 24 & 60 & 7.4 \\
\hline 2 & $R / L^{*} F$ sup medial gyrus & 2 & 28 & 59 & 7.9 \\
\hline 3 & L F sup gyrus & -22 & 23 & 61 & 7.7 \\
\hline 4 & R F middle gyrus & 37 & 30 & 50 & 6.9 \\
\hline 5 & R F sup gyrus & 13 & 40 & 54 & 6.9 \\
\hline 6 & L F sup gyrus & -12 & 41 & 54 & 6.8 \\
\hline 7 & L F middle gyrus & -35 & 30 & 50 & 7.2 \\
\hline 8 & R F sup gyrus & 25 & 47 & 44 & 6.1 \\
\hline 9 & $R / L^{*} F$ sup medial gyrus & 2 & 50 & 44 & 6.7 \\
\hline 10 & L F sup gyrus & -22 & 47 & 43 & 6.7 \\
\hline 11 & R F middle gyrus & 38 & 52 & 29 & 5.6 \\
\hline 12 & R F sup gyrus & 14 & 61 & 34 & 6.1 \\
\hline 13 & LF sup gyrus & -12 & 60 & 35 & 5.7 \\
\hline 14 & L F middle gyrus & -36 & 51 & 29 & 6.4 \\
\hline 15 & R F sup gyrus & 26 & 65 & 19 & 4.9 \\
\hline 16 & $R / L^{*} F$ sup medial gyrus & 3 & 66 & 22 & 7.1 \\
\hline 17 & L F sup gyrus & -24 & 65 & 20 & 5.6 \\
\hline 18 & $\mathrm{R} F$ middle gyrus & 39 & 63 & 4 & 4.9 \\
\hline 19 & R F sup gyrus & 15 & 71 & 9 & 4.5 \\
\hline 20 & L F sup gyrus & -13 & 72 & 9 & 4.9 \\
\hline 21 & L F middle gyrus & -37 & 63 & 4 & 5.1 \\
\hline 22 & R F sup orb gyrus & 28 & 68 & -5 & 4.1 \\
\hline 23 & $\mathrm{R} / \mathrm{L}^{*} \mathrm{~F}$ middle orb gyrus & 3 & 68 & -4 & 5.4 \\
\hline 24 & L F sup orb gyrus & -24 & 68 & -5 & 4.2 \\
\hline
\end{tabular}

$R$, right; $L$, left; F, frontal; Orb, orbital; sup, superior.

${ }^{*}$ Channels located on the nasion-Inion virtual line.

removed by bandpass filtering between 0.02 and $1 \mathrm{~Hz}$ and, in order to prevent movement artifacts, a further filter was used to remove detections with rapid changes in $\mathrm{HbO} 2$ concentration (signal variations $>0.1 \mathrm{mM} \bullet \mathrm{mm}$ over two consecutive samples). We also visually checked the signals recorded in each channel of all subjects, in order to detect low signal-to-noise ratio due to bad transmission of near-infrared light (e.g., due to hair obstruction). There was no need to exclude any registrations from data analysis due to a bad signal-to-noise ratio.

An event design was used and the active channels were identified by means of paired $t$-tests. In each participant, for every channel, an arbitrary baseline was calculated as the mean in relative changes of $\mathrm{HbO} 2$ in the $2 \mathrm{~s}$ before the onset of the green slide. The hemodynamic response associated with each choice was calculated as the mean change in $\mathrm{HbO} 2$ concentration over the $10 \mathrm{~s}$ after the onset. To identify the activated channels, e.g., those showing a $\mathrm{HbO} 2$ increase, we performed one-tailed paired $t$-tests and compared, for each channel, the baseline and the choice associated hemodynamic response. In order to evidence possible changes in DLPFC activation associated with different choice risk levels, as the performance went on, the time course of task execution was divided in four 
periods, each one consisting of 25 choices, and DLPFC activation was distinctly analyzed for low and high risk choices in each period.

To control Type I error in multiple testing situations, we used a false discovery rate (FDR) approach (Genovese et al., 2002; Singh and Dan, 2006), that controls the proportion of false positives among channels that are significantly detected. We selected a $q$ value of 0.05 , so that there were no more than $5 \%$ false positives (on average) in the number of channels emerging with significant contrasts. Statistical analyses were conducted using SPSS version 13.0 for Windows (SPSS Inc., Chicago, IL, USA).

\section{RESULTS}

All but one participant in our sample chose advantageously, showing an IGT net score above the cut-off threshold of at least 50 choices from advantageous decks. Table 2 reports single performances.

During the first task period (1st-25th choice), five channels passed the FDR threshold $(P<$ FDR 0.05$)$ in association with low risk choices (advantageous card decks $\mathrm{C}$ and D). They were

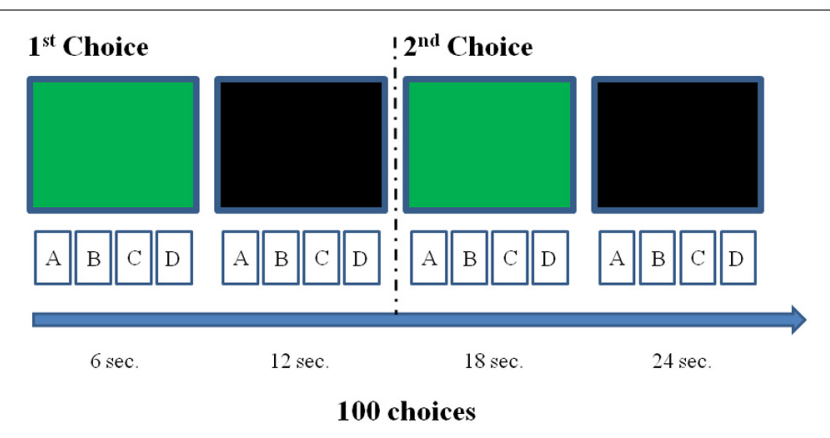

FIGURE 2 | Illustration of the experimental procedure concerning the first two choices. Each choice could be made just after the presentation of the green slide, but within $12 \mathrm{~s}$ (e.g., the interval between choices we selected to allow the detection of a complete hemodynamic response associated with each decision). The black screen was inserted to fill the $12 \mathrm{~s}$ inter-choice interval creating an alternation with the green slide, which had to be again presented to allow the next choice. This was repeated for 100 times.

Table 2 | List of IGT participants' performances (in bold it is reported the one with a score under the cut-off threshold of at least $\mathbf{5 0}$ advantageous choices).

\begin{tabular}{llll}
\hline Participant id & Sex & Age & Igt score \\
\hline 1 & M & 26 & 76 \\
2 & F & 24 & 58 \\
3 & F & 25 & 56 \\
$\mathbf{4}$ & F & $\mathbf{2 6}$ & $\mathbf{3 6}$ \\
5 & M & 27 & 84 \\
6 & M & 35 & 56 \\
7 & F & 35 & 78 \\
8 & F & 26 & 86 \\
9 & M & 21 & 78 \\
10 & F & 38 & 66 \\
11 & F & 22 & 62
\end{tabular}

channel $13\left(t_{10}=-2.818 ; P=0.009\right)$, located on left superior frontal gyrus, channel $15\left[t_{(10)}=-6.471 ; P<0.001\right]$, located on right superior frontal gyrus, channel $16\left[t_{(10)}=-3.454 ; P=\right.$ $0.003]$, located on right/left superior and medial frontal gyrus, channel $19\left[t_{(10)}=-3.042 ; P=0.006\right]$, located on right superior frontal gyrus, and channel $22\left[t_{(10)}=-3.222 ; P=0.0045\right]$, located on right superior/orbital frontal gyrus (Figures 3, 4). Instead, in association with high risk choices (disadvantageous card decks $\mathrm{A}$ and $\mathrm{B}$ ), no channel passed the FDR threshold.

During the second task period (26th-50th choice), in association with low risk choices, only channel $19\left[t_{(10)}=-3.910\right.$; $P=0.0015]$, located on right superior frontal gyrus, passed the FDR threshold. In association with high risk choices, on the other hand, eight channels passed the FDR threshold $\left(P<\right.$ FDR 0.05). They were channel $2\left[t_{(10)}=-5.858\right.$; $P=0.006]$, located on right/left superior and medial frontal gyrus, channel $3\left[t_{(10)}=-4.300 ; P=0.001\right]$, located on left superior frontal gyrus, channel $9\left[t_{(10)}=-3.841 ; P=0.0015\right]$, located on right/left superior and medial frontal gyrus, channel $12\left[t_{(10)}=-3.441 ; P=0.003\right]$, located on right superior frontal gyrus, channel $13\left[t_{(10)}=-3.349 ; P=0.0035\right]$, located on left superior frontal gyrus, channel $15\left[t_{(10)}=-4.078\right.$; $P=0.001]$, located on right superior frontal gyrus, channel $16\left[t_{(10)}=-2.688 ; P=0.0115\right]$, located on right/left superior and medial frontal gyrus, and channel $19\left[t_{(10)}=-4.382 ; P=\right.$ $0.0005]$, located on right superior frontal gyrus (Figures 5, 6).

During the third and fourth task period (51st-100th choice), no channel passed the FDR threshold ( $P<$ FDR 0.05), for either low- or high-risk choices.

Four channels were activated in association with both low- or high-risk choices. They were channels $13,15,16$, and 19, covering left, right and central portions of DLPFC. Additionally, in association with low-risk choices, also the right frontal pole was activated (channel 22), while, in association with high-risk choices, the DLPFC activation extended more posteriorly, to channels 2, 3, and 9.

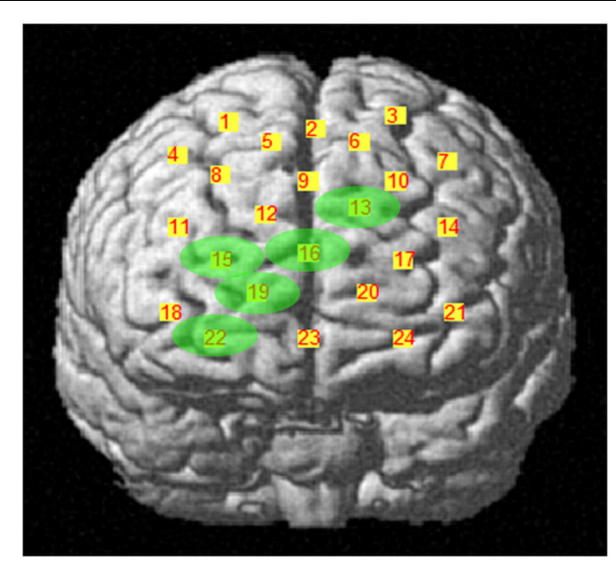

FIGURE 3 | Cortical location, on a rendered brain, of channels found significantly activated in association with low risk choices (evidence in green), during the first IGT period. 


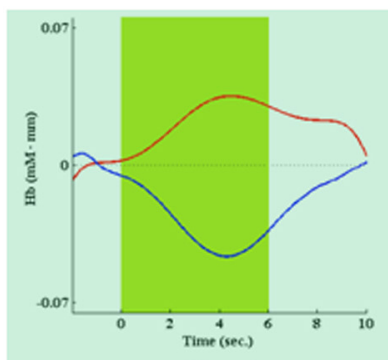

Ch 13

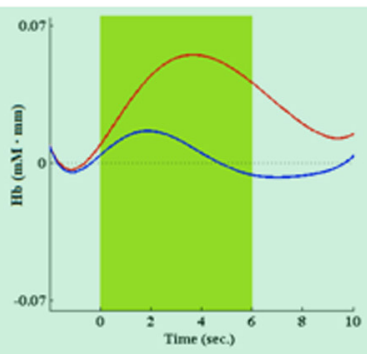

Ch 15

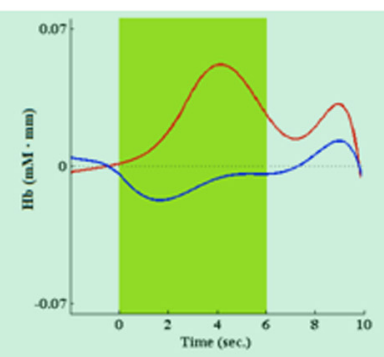

Ch 16

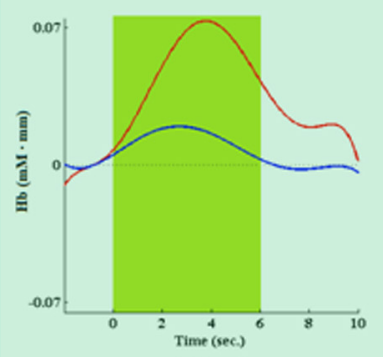

Ch 19

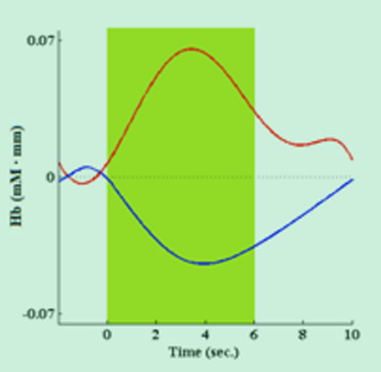

Ch 22
FIGURE 4 | Time courses of $\mathrm{HbO2}$ (in red) and deoxy-hemoglobin (in blue) concentration changes, reported in $\mathrm{mMemm}$, in channels found significantly activated during the first IGT period, in association with low risk choices. The dark green area shows when the green slide was presented on a computer screen to participants, signaling that the choice could be made.

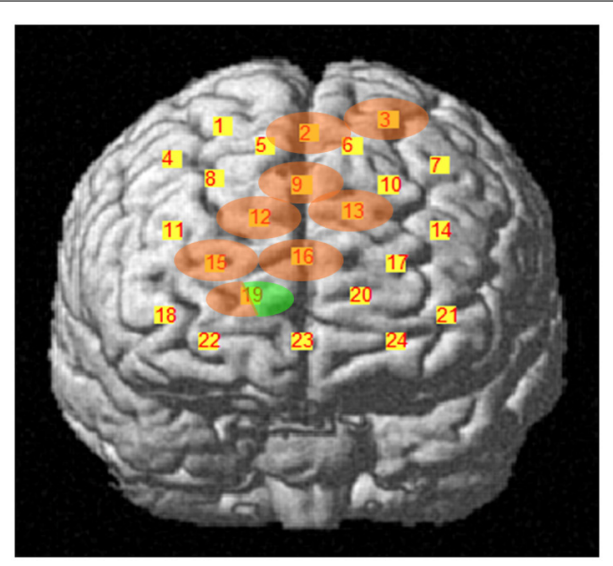

FIGURE 5 | Cortical location, on a rendered brain, of channels found significantly activated in association with high risk choices (evidence in pink), or in high and low risk choices (evidence in pink/green), during the second task period.

\section{DISCUSSION}

We used multichannel NIRS to study if DLPFC is differentially activated, over time, by low or high risk choices during the execution of the complete administration of IGT, a playing card task developed to study DM under uncertainty. We have found that DLPFC significantly contributes to the IGT execution in the first half of the task. Additionally, we found different time courses in its activation as the task went on, associated with low or high risk choices. Specifically, DLPFC was shown to be activated by low risk choices in the first period (1st-25th choice) and by high risk choices in the second period (26th-50th choice) of IGT performance. Activated DLPFC areas associated with low or high risk choices partially overlapped in both left, right and central portions of the DLPFC region monitored. In association with low risk choices (first period), along with activation of left/right superior frontal gyrus and left/right medial frontal gyrus (channels 13, 15, 16 , and 19), also the right frontal pole was activated (channel 22). In association with high risk choices (second period), the DLPFC activation was more extensive and involved more posterior areas, possibly including the supplementary motor area (channels 2 and 3 ). In both cases, activation was slightly lateralized on the right side. No significant variations in DLPFC activity emerged during the second half of IGT execution, regardless of choice risk level.

The involvement of DLPFC, especially with a right lateralization, in IGT execution has already been shown. Clinically, a bad IGT performance was observed in patients with right DLPFC lesions (Clark et al., 2003; Fellows and Farah, 2005) and, by neuroimaging, DLPFC cortical areas were found activated during the IGT test (Ernst et al., 2002, 2003; Bolla et al., 2003, 2005). Besides DLPFC, other areas may play a role in DM, such as VMPFC (Bechara et al., 2000a), the amygdala (Bechara, 2001; Bar-On et al., 2003) and the insula (Lin et al., 2008; Lawrence et al., 2009), but such areas are too deeply located to be detected by NIRS devices. Primary and secondary sensory areas (Bechara, 2001; Bar-On et al., 2003) are involved in DM as well, but they were outside our region of interest. More recently, Hartstra et al. (2010) have demonstrated, by fMRI, that activation of DLPFC during 


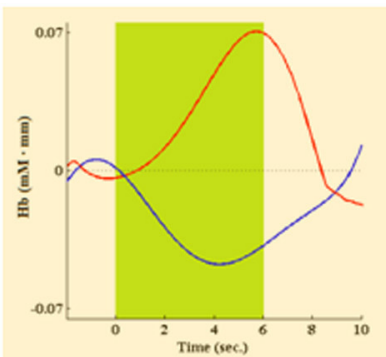

Ch 2

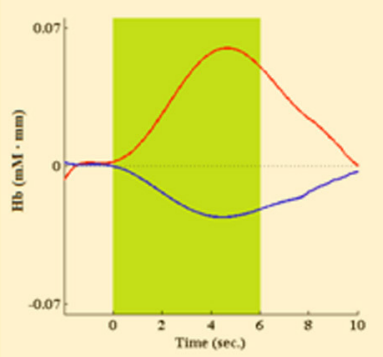

Ch 12

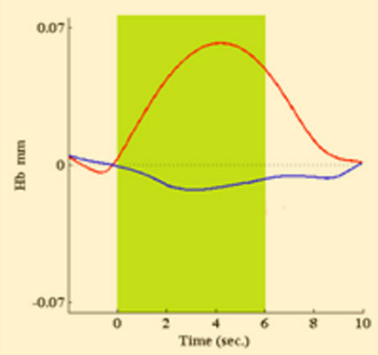

Ch 16

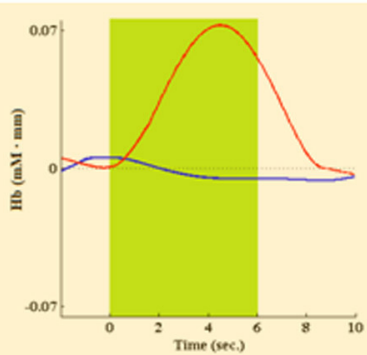

Ch 3

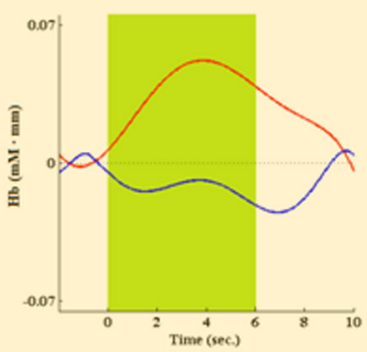

Ch 13

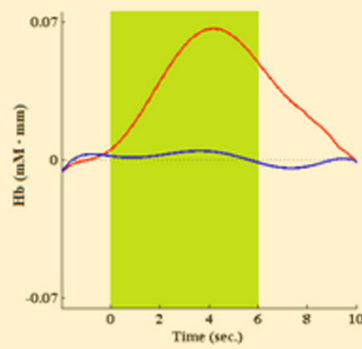

Ch 19

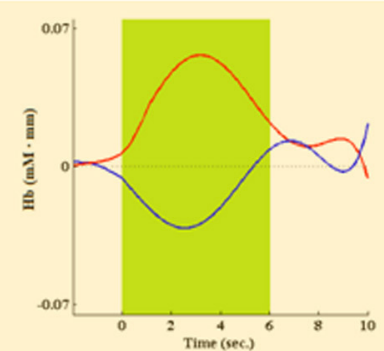

Ch 9

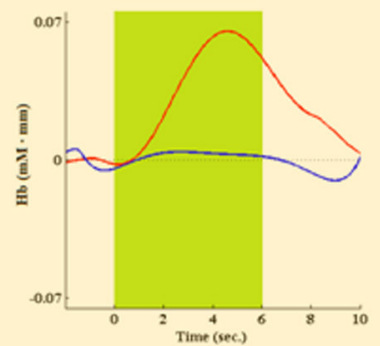

Ch 15

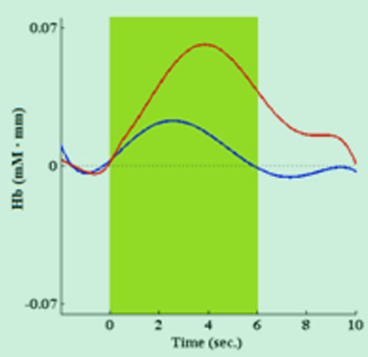

Ch 19
FIGURE 6 | Time courses of $\mathrm{HbO2}$ (in red) and deoxy-hemoglobin (in blue) concentration changes, reported in $\mathrm{mM \bullet mm}$, in channels found significantly activated during the second IGT period, in association with high risk choices (pink background) or low risk choices (green background). The green area shows when the green slide was presented on a computer screen to participants, signaling that the choice could be made.
IGT execution changes over time: it is significant in the first part of the modified task they used and becomes non-significant later. These authors speculate that earlier activation observed in DLPFC may be attributed to the involvement of working memory, which would be necessary for active learning of task rules (see also Bechara et al., 1998; Clark et al., 2003; Fellows and Farah, 2005; Martinez-Selva et al., 2006). In addition, they hypothesize that later med-OFC activation is associated with the representation of learned reward values, guiding successive choices. In agreement with the previous study, we showed an early significant involvement of DLPFC in IGT performance as well, but we found a differential activation according to choice risk level. DLPFC was activated earlier by low risk choices and later by high risk choices. Study designs differed, as Hartstra et al. (2010) aimed to study the role of prefrontal cortex in rules learning, while we aimed to show the DLPFC contribution to IGT performance in the setting of high or low risk choices. In this regard, a meta-analysis of fMRI studies has demonstrated that risk-related
DLPFC activation occurs when a choice is involved (Mohr et al., 2010). Moreover, it has been recently found that DLPFC mediates the discrimination of IGT disadvantageous choices (Christakou et al., 2009), and the activation intensity in such region has been positively correlated with task performance (Lawrence et al., 2009).

Our results on the differential DLPFC activation over time, in association with low or high risk choices during the IGT, may seem to indicate that working memory is not the only neurocognitive resource supplied by DLPFC. We speculate that two superior cognitive functions, mediated by DLPFC as well, may explain our observations: attention shifting and response inhibition. It is worth noting that, in IGT, high risk choices are those with a greater immediate reward, but with a delayed greater loss. On the contrary, low risk choices are associated with lower immediate rewards, but with a delayed gain. DLPFC activation showed by our participants seems to indicate a shift in attention, as the task went on. Their attention shifted from the decks with lower 
immediate rewards, in the first task period (1st-25th choice), to those with greater losses, in the second task period (26th50 th choice). In both cases, an attention response, mediated by DLPFC (e.g., Manes et al., 2002), was elicited by those decks (and related choices) that seemed to be more disadvantageous (see also Christakou et al., 2009; Lawrence et al., 2009). Moreover, in the second period of the task, when DLPFC was activated in association with high risk choices, frontocentral cortical areas (channels 9 and 16) and the supplementary motor area (channels 2 and 3) were also activated. Both regions have been associated with response inhibition and behavioral control (Elliott et al., 2000; van Gaal et al., 2008; Fassbender et al., 2009).

Our experimental procedure aimed to assess DLPFC activation associated with low or high risk choices, as they were made. Consequently, the activation of cortical regions with a role in response inhibition was detected when the high-risk decision was made. We speculate that the extended DLPFC activation in the second IGT quarter, may reflect a conflict between previously learned and newer rules, in order to make the best decision. The activation involving the right frontal pole in the first quarter may indicate an attempt to elaborate more abstract task rules (e.g., Venkatraman and Huettel, 2012), as an initial approach to an unknown complex task. Concerning the absence of DLPFC activation in the third and fourth period of the IGT, we speculate that it may be due to VMPFC taking over the task, which is undetectable by fNIRS. Indirectly, this hypothesis is supported by Hartstra et al. (2010), who found only a later involvement of medial prefrontal cortex regions (med-OFC in their case) in IGT performance. Moreover, Lawrence et al. (2009) found a linear decrease in lateral OFC and pre-supplementary motor area activation as IGT execution progressed.

Our study has some obvious limitations. First, we enrolled a limited number of subjects and it should be considered a pilot study. Although functional neuroimaging studies are typically performed in small populations, our results need to be confirmed in a larger sample. Characterizing participants for personality traits and mood state would further improve such a study, since it has been proved that these variables have an influence on IGT performance (Buelow and Suhr, 2009). Second, multichannel NIRS has a poorer spatial resolution than fMRI. However, previous research has shown a good correlation between these two techniques (Toronov et al., 2001; Strangman et al., 2002). Third, NIRS measurement of hemoglobin variations are limited to the lateral surface of the cerebral cortex. Fourth, the experimental design we chose did not allow us to discriminate among the specific prefrontal functions involved in the early distinction between low- and high-risk choices. Finally, some possible systemic effects we have not directly checked, e.g., blood flow variation in the scalp during the experiment, could have had an influence on our results. However, this was probably not the case because an asymmetric pattern of cortical activation was observed.

In conclusion, this study has further deepened the complex role of DLPFC to early DM under uncertainty process, as assessed by IGT. We have shown that, in the first half of the performance, DLPFC activation differs over time, according to the risk level of the choice. We propose that, beside working memory, other complex functions, such as attention shifting and response inhibition, are involved in sustaining the DLPFC role in discriminating disadvantageous choices during the task. Thereafter, as rule learning establishes, DM under uncertainty seems to be prevalently mediated by VMPFC. Thus, it emerges a wide, complex and temporally dynamic involvement of the prefrontal cortex in decisions concerning the ordinary uncertainty of everyday life.

\section{ACKNOWLEDGMENT}

This study has been financed by the grant N. 50/11 of the Institute for Maternal and Child Health-IRCCS "Burlo Garofolo"Trieste, Italy.

\section{REFERENCES}

Bar-On, R., Tranel, D., Denburg, N. L., and Bechara, A. (2003). Exploring the neurological substrate of emotional and social intelligence. Brain 126, 1790-1800. doi: 10.1093/brain/awg177

Bechara, A. (2001). Neurobiology of decision-making: risk and reward. Semin. Clin. Neuropsychiatry 6, 205-216. doi: 10.1053/scnp.2001.22927

Bechara, A., Damasio, A. R., Damasio, H., and Anderson, S. W. (1994). Insensitivity to the future consequences following damage to prefrontal cortex. Cognition 50, 7-15. doi: 10.1016/0010-0277(94)90018-3

Bechara, A., Damasio, H., and Damasio, A. R. (2000b). Emotion, decision making and the orbitofrontal cortex. Cerb. Cortex 10, 295-307. doi: 10.1093/cercor/10.3.295

Bechara, A., Damasio, H., Damasio, A. R., and Lee, G. P. (1999). Different contributions of the human amygdala and ventromedial prefrontal cortex to decision-making. J. Neurosci. 19, 5473-5481.

Bechara, A., Damasio, H., Tranel, D., and Anderson, S. W. (1998). Dissociation of working memory from decision-making within the human prefrontal cortex. J. Neurosci. 18, 428-437.

Bechara, A., Tranel, D., and Damasio, H. (2000a). Charachterization of the decision-making deficit of patients with ventromedial prefrontal cortex lesions. Brain 123, 2189-2202. doi: 10.1093/brain/123.11.2189

Bolla, K. I., Eldreth, D. A., London, E. D., Kiehl, K. A., Mouratidis, M., Contoreggi, C., et al. (2003). Orbitofrontal cortex dysfunction in abstinent cocaine abusers performing a decision-making task. Neuroimage 19, 1085-1094. doi: 10.1016/S1053-8119(03)00113-7

Bolla, K. I., Eldreth, D. A., Matochik, J. A., and Cadet J. L. (2005). Neural substrates of faulty decision-making in abstinent marijuana users. Neuroimage 26, 480-492. doi: 10.1016/j.neuroimage.2005.02.012

Buelow, M. T., and Suhr, J. A. (2009). Construct validity of the Iowa Gambling Task. Neurpsychol. Rev. 19, 102-114. doi: 10.1007/s11065-009-9083-4

Cazzell, M., Li, L., Lin, Z. J., Petel, S. J., and Liu, H. (2012). Comparison of neuronal correlates of risk decision making between genders: an exploratory fNIRS study of the Balloon Analogue Risk Task (BART). Neuroimage 62, 1869-1911. doi: 10.1016/j.neuroimage.2012.05.030

Christakou, A., Brammer, M., Giampietro, V., and Rubia, K. (2009). Right ventromedial and dorsolateral prefrontal cortices mediate adaptive decisions under ambiguity by integrating choice utility and outcome evaluation. J. Neurosci. 29, 11020-11028. doi: 10.1523/JNEUROSCI.1279-09.2009

Clark, L., Manes, F., Antoun, N., Sahakian, B. J., and Robbins, T. W. (2003). The contributions of lesion laterality and lesion volume to decision-making impairment following forntal lobe damage. Neuropsychologia 41, 1474-1483. doi: 10.1016/S0028-3932(03)00081-2

Damasio, A. R. (1994). Descartes' Error: Emotion, Reason, and The Human Brain. New York, NY: Putnam.

Elliott, R., Dolan, R. J., and Frith C. D. (2000). Dissociable functions in the medial and lateral orbitofrontal cortex: evidence from human neuroimaging studies. Cereb. Cortex 10, 308-317. doi: 10.1093/cercor/10.3.308

Ernst, M., Bolla, K., Mouratidis, M., Contoreggi, C., Matochik, J. A., Kurian, V., et al. (2002). Decision-making in a risk-taking task: a PET study. Neuropsychopharmachology 26, 682-691. doi: 10.1016/S0893-133X(01)00414-6

Ernst, M., Kimes, A. S., London, E. D., Matochik, J. A., Eldreth, D., Tata, S., et al. (2003). Neural substrates of decision making in adults with attention deficit hyperactivity disorder. Am. J. Psychiatry 160, 1061-1070. doi: 10.1176/appi.ajp.160.6.1061 
Fassbender, C., Hester, R., Murphy, K., Foxe, J. J., Foxe, D. M., and Garavan, H. (2009). Prefrontal and midline interactions mediating behavioural control. Eur. J. Neurosci. 29, 181-187. doi: 10.1111/j.1460-9568.2008.06557.x

Fellows, L. K., and Farah, M. J. (2005). Different underlying impairments in decision-making following ventromedial and dorsolateral frontal lobe damage in humans. Cereb. Cortex 15, 58-63. doi: 10.1093/cercor/bhh108

Genovese, C. R., Lazar, N. A., and Nichols, T. (2002). Thresholding of statistical maps in functional neuroimaging using the false discovery rate. Neuroimage 15 , 870-878. doi: 10.1006/nimg.2001.1037

Gläscher, J., Adolphs, R., Damasio, H., Bechara, A., Rudrauf, D., Clamia, M., et al. (2012). Lesion mapping of cognitive control and value-based decision making in the prefrontal cortex. Proc. Natl. Acad. Sci. U.S.A. 109, 14681-14686. doi: 10.1073/pnas.1206608109

Gupta, R., Duff, M. C., Denburg, N. L., Cohen, N. J., Bechara, A., and Tranel, D. (2009). Declarative memory is critical for sustained advantageous complex decision-making. Neuropsychologia 47, 1686-1693. doi: 10.1016/j.neuropsychologia.2009.02.007

Hartstra, E., Oldenburg, J. F., Van Leijenhorst, L., Rombouts, S. A., and Crone, E. A. (2010). Brain regions involved in the learning and application of reward rules in a two-deck gambling task. Neuropsychologia 48, 1438-1446. doi: 10.1016/j.neuropsychologia.2010.01.012

Ito, Y., Kennan, R. P., Watanabe, E., and Koizumi, H. (2000). Assessment of heating effects in skin during continuous wave near infrared spectroscopy. J. Biomed. Opt. 5, 383-390. doi: 10.1117/1.1287730

Jasper, H. H. (1958). The ten- twenty electrode system of the International Federation. Electroencephalogr. Clin. Neurophysiol. 10, 367-380.

Lawrence, N. S., Jollant, F., O’Daly, O., Zelaya, F., and Phillips, M. L. (2009). Distinct roles of prefrontal cortical subregions in the Iowa Gambling Task. Cereb. Cortex 19, 1134-1143. doi: 10.1093/cercor/bhn154

Lin, C. H., Chiu, Y. C., Cheng, C. M., and Hsieh, J. C. (2008). Brain maps of Iowa gambling task. BMC Neurosci. 9:72. doi: 10.1186/1471-2202-9-72

Maki, A., Yamashita, Y., Ito, Y., Watanabe, F., Mayanagi, Y., and Koizumi, H. (1995). Spatial and temporal analysis of human motor activity using non-invasive NIR topography. Med. Phys. 22, 1997-2005. doi: 10.1118/1. 597496

Manes, F., Sahakian, B., Clark, L., Rogers, R., Antoun, N., Aitken, M., et al. (2002). Decision-making processes following damage to prefrontal cortex. Brain 125, 624-639. doi: 10.1093/brain/awf049

Martinez-Selva, J. M., Sanchez-Navarro, J. P., Bechara, A., and Roman, F. (2006). Brain mechanisms involved in decision-making. Rev. Neurol. 42, 411-418.

Meek, J. (2002). Basic principles of optical imaging and application to the study of infant development. Dev. Sci. 5, 371-380. doi: 10.1111/1467-7687. 00376

Mohr, P. N. C., Biele, G., and Heekeren, H. R. (2010). Neural processing of risk. J. Neurosci. 30, 6613-6619. doi: 10.1523/JNEUROSCI.0003-10.2010

Oldfield, R. C. (1971). The assessment and analysis of handedness: the Endinburg inventory. Neuropsychologia 9, 97-113. doi: 10.1016/0028-3932(71)90067-4

Oya, H., Adolphs, R., Kawasaki, H., Bechara, A., Damasio, A., and Howard, M. A. 3rd. (2005). Electrophysiological correlates of reward prediction error recorded in the human prefrontal cortex. Proc. Natl. Acad. Sci. U.S.A. 102, 8351-8356. doi: 10.1073/pnas.0500899102

Schroeter, M. L., Zysset, S., and von Cramon, D. Y. (2004). Shortening intertrial intervals in event-related cognitive studies with near-infrared spectroscopy. Neuroimage 22, 341-346. doi: 10.1016/j.neuroimage.2003.12.041

Singh, A. K., and Dan, I. (2006). Exploring the false discovery rate in multichannel NIRS. Neuroimage 33, 542-549. doi: 10.1016/j.neuroimage.2006.06.047
Singh, A., K., Okamoto, M., Dan, H., Jurcak, V., and Dan, I. (2005). Spatial registration of multichannel multi-subject fNIRS data to MNI space without MRI. Neuroimage 27, 842-851. doi: 10.1016/j.neuroimage.2005.05.019

Strangman, G., Culver, J. B., Thompson, J. H., and Boas, D. A. (2002). A quantitative comparison of simultaneous BOLD fMRI and NIRS recordings during functional brain activation. Neuroimage 17, 719-731. doi: 10.1006/nimg.2002.1227

Stocco, A., and Fum, D. (2007). Implicit emotional biases in decision making: the case of the Iowa gambling task. Brain Cogn. 66, 253-259. doi: 10.1016/j.bandc.2007.09.002

Taga, G., Asakawa, K., Maki, A., Konishi, Y., and Koizumi, H. (2003). Brain imaging in awake infants by near-infrared optical topography. Proc. Natl. Acad. Sci. U.S.A. 19, 10722-10727. doi: 10.1073/pnas.1932552100

Toronov, V., Webb, A., Choi, J. H., Wolf, M., Michalos, A., Gratton, E., et al. (2001). Investigation of human brain hemodynamics by simultaneous nearinfrared spectroscopy and functional magnetic resonance imaging. Med. Phys. 28, 521-527. doi: 10.1118/1.1354627

Tsuzuki, D., Jurcak V., Singh, A. K., Okamoto M., Watanabe W., and Dan I. (2007). Virtual spatial registration of stand-alone fNIRS data to MNI space. Neuroimage 34, 1506-1518. doi: 10.1016/j.neuroimage.2006.10.043

Tzourio-Mazoyer, N., Landeau, B., Papathanassiou, D., Crivello, F., Etard, O., Delcroix, N., et al. (2002). Automated anatomical labelling of activation in SPM using a macroscopic anatomical parcellation of the MNI MRI single-subject brain. Neuroimage 15, 273-289. doi: 10.1006/nimg.2001.0978

van Gaal, S., Ridderinkhof, K. R., Fahrenfort, J. J., Scholte, H. S., and Lamme, V. A. F. (2008). Frontal cortex mediates unconsciously triggered inhibitory control. J. Neurosci. 28, 8053-8062. doi: 10.1523/JNEUROSCI.1278-08.2008

Venkatraman, V., and Huettel, S. A. (2012). Strategic control in decisionmaking under uncertinity. Eur. J. Neurosci. 35, 1075-7082. doi: 10.1111/j.14609568.2012.08009.x

Wise, S. P. (2008). Forward frontal fields: phylogeny and fundamental function. Trends Neurosci. 31, 599-608. doi: 10.1016/j.tins.2008.08.008

Wobst, P., Wenzel, R., Kohl, M., Obrig, H., and Villringer, A. (2001). Linear aspects of changes in deoxygenated hemoglobin concentration and cytochrome oxidase oxidation during brain activation. Neuroimage 13, 520-530. doi: 10.1006/nimg.2000.0706

Ye, J. C., Tak, S. H., Jang, K. E., Jung, J. W., and Jang, J. D. (2009). NIRS-SPM: statistical parametric mapping for near-infrared spectroscopy. Neuroimage 44, 428-447. doi: 10.1016/j.neuroimage.2008.08.036

Conflict of Interest Statement: The authors declare that the research was conducted in the absence of any commercial or financial relationships that could be construed as a potential conflict of interest.

Received: 30 September 2013; accepted: 06 June 2014; published online: 24 June 2014. Citation: Bembich S, Clarici A, Vecchiet C, Baldassi G, Cont G and Demarini S (2014) Differences in time course activation of dorsolateral prefrontal cortex associated with low or high risk choices in a gambling task. Front. Hum. Neurosci. 8:464. doi: 10.3389/ fnhum.2014.00464

This article was submitted to the journal Frontiers in Human Neuroscience.

Copyright (c) 2014 Bembich, Clarici, Vecchiet, Baldassi, Cont and Demarini. This is an open-access article distributed under the terms of the Creative Commons Attribution License (CC BY). The use, distribution or reproduction in other forums is permitted, provided the original author(s) or licensor are credited and that the original publication in this journal is cited, in accordance with accepted academic practice. No use, distribution or reproduction is permitted which does not comply with these terms. 